\title{
The palatine tonsil bacteriome, but not the mycobiome, is altered in HIV infection
}

\author{
Yuto Fukui ${ }^{1,2^{*}}$, Kotaro Aoki ${ }^{1}$, Yoshikazu Ishii ${ }^{1}$ and Kazuhiro Tateda ${ }^{1}$
}

\begin{abstract}
Background: Microbial flora in several organs of HIV-infected individuals have been characterized; however, the palatine tonsil bacteriome and mycobiome and their relationship with each other remain unclear. Determining the palatine tonsil microbiome may provide a better understanding of the pathogenesis of oral and systemic complications in HIV-infected individuals. We conducted a cross-sectional study to characterize the palatine tonsil microbiome in HIV-infected individuals.

Results: Palatine tonsillar swabs were collected from 46 HIV-infected and 20 HIV-uninfected individuals. The bacteriome and mycobiome were analyzed by amplicon sequencing using Illumina MiSeq. The palatine tonsil bacteriome of the HIV-infected individuals differed from that of HIV-uninfected individuals in terms of the decreased relative abundances of the commensal genera Neisseria and Haemophilus. At the species level, the relative abundances and presence of Capnocytophaga ochracea, Neisseria cinerea, and Selenomonas noxia were higher in the HIV-infected group than those in the HIV-uninfected group. In contrast, fungal diversity and composition did not differ significantly between the two groups. Microbial intercorrelation analysis revealed that Candida and Neisseria were negatively correlated with each other in the HIV-infected group. HIV immune status did not influence the palatine tonsil microbiome in the HIV-infected individuals.
\end{abstract}

Conclusions: HIV-infected individuals exhibit dysbiotic changes in their palatine tonsil bacteriome, independent of immunological status.

Keywords: Human immunodeficiency virus, Palatine tonsil microbiome, Bacteriome, Mycobiome

\section{Background}

Human immunodeficiency virus (HIV) infection is characterized by progressively reduced cell-mediated immunity, as reflected by the $\mathrm{CD}^{+}{ }^{+} \mathrm{T}$-cell count, resulting in increased opportunistic infections [1]. Antiretroviral therapy (ART) has increased the life expectancy of HIV-infected individuals, accompanied by improved cellular immunity and reduced frequency of diseases associated with acquired immunodeficiency syndrome (AIDS) [2, 3]. Despite the successful use of ART, HIV-infected individuals continue to experience excessive morbidity and mortality from non-AIDS defining infectious diseases and noninfectious chronic comorbidities $[4,5]$.

\footnotetext{
* Correspondence: yuuto.fukui@med.toho-u.ac.jp

1 Department of Microbiology and Infectious Diseases, Toho University Graduate School of Medicine, 5-21-16 Omorinishi, Ota-ku, Tokyo 143-8540, Japan

${ }^{2}$ Department of Infectious Diseases, Toho University Omori Medical Center, 6-11-1 Omorinishi, Ota-ku, Tokyo 143-8541, Japan
}

The microbiome (the collective genomes of microbial flora) of HIV-infected individuals is gradually being elucidated [6, 7], as human microbiome research progresses [8]. The role of microbial flora in pathogenesis of HIV infection has been the subject of much research in recent years [7, 9-13]. Dysbiosis during HIV infection such as increased abundance of pathogenic Prevotella in the oral cavity and decreased abundance of Lactobacillus in the gut has been reported, and the loss of a normal microbial flora may influence HIV transmission, prevention, progression and prognosis [12, 14-20]. However, most studies that have characterized the human microbiome in health and disease, including in HIV infection, have focused only on the bacteriome [21]. Fungi are also important in the human microbiome [22]; thus, more research is needed on the mycobiome and its relationships with the bacteriome.

The human palatine tonsils are mucosa-associated lymphoid tissues (MALT) located in the oropharynx and

(c) The Author(s). 2018 Open Access This article is distributed under the terms of the Creative Commons Attribution 4.0 International License (http://creativecommons.org/licenses/by/4.0/), which permits unrestricted use, distribution, and 
provide mucosal protection [23]. Based on their location, the palatine tonsils are among the early sites that encounter microbial and environmental antigens in the human body [23, 24]. Therefore, the palatine tonsil microbiome of HIV-infected individuals may play a key role in opportunistic infections; however, this remains to be investigated.

We conducted a cross-sectional study to characterize the palatine tonsil bacteriome, mycobiome and their intercorrelations with each other among HIV-infected individuals.

\section{Results}

\section{Participants characteristics}

Forty-six HIV-infected and 20 HIV-uninfected participants were enrolled. No differences in age, sex, smoking status, or white blood cell (WBC) count were noted between the groups (Table 1). No individuals had severe periodontal diseases that required treatment. The median and nadir $\mathrm{CD} 4^{+}$T-cell count of the HIV-infected group was 438 cells $/ \mu \mathrm{L}$ (interquartile range [IQR] 274618) and 164 cells/ $\mu \mathrm{L}$ (IQR 94-306), including 6 severely immunocompromised individuals (13\%) with $\mathrm{CD}^{+}$ T-cell counts below 200 cells $/ \mu \mathrm{L}$. The median plasma viral load of the HIV-infected group was 0 copy $/ \mathrm{mL}$ (IQR 0-4505), with 13 individuals having detectable viremia (viral load $>200$ copies $/ \mathrm{mL}$ ). Thirty-four of the 46 HIV-infected individuals (74\%) received ART.

\section{HIV-infected individuals had an altered palatine tonsil bacteriome}

One HIV-infected sample did not contain sufficient reads for analysis; therefore, $45 \mathrm{HIV}$-infected and $20 \mathrm{HIV}$ - uninfected participants were included in the bacterial analysis. We first assessed the alpha diversity (the number of observed operational taxonomy units [OTUs], Shannon entropy and phylogenetic diversity) of the palatine tonsil bacteriome from 14,737 reads. The HIV-infected individuals had a significantly increased number of observed OTUs (Additional file 1: Table S1). Beta diversity, determined by principal coordinates analysis using weighted UniFrac distance metrics, showed that the HIV-infected palatine tonsil bacteriome was distinct from that of the HIV-uninfected individuals $(p=$ 0.02 , permutational multivariate analysis of variance [PERMANOVA]) (Additional file 2: Figure S1).

To determine which taxa differed between the HIV-infected and HIV-uninfected individuals, we compared the relative abundances between them at different taxonomic levels.

Phylum-level analysis revealed that five phyla (Firmicutes, Bacteroidetes, Proteobacteria, Fusobacteria, Actinobacteria) were abundant, and Firmicutes was the most abundant in both groups (Additional file 1: Table S2). Compared to the HIV-uninfected individuals, the HIV-infected individuals had a significantly increased relative abundance of Firmicutes $(p=0.009)$ and significantly decreased relative abundances of Proteobacteria $(p=0.04)$ and Fusobacteria $(p=0.03)$.

A total of 82 genera were detected in both groups. At the genus level, we identified the core bacteriome of each group, which was defined as the genera present in $100 \%$ of samples [25]. The core palatine tonsil bacteriome consisted of 18 genera in both groups, of which 16 genera (Streptococcus, Prevotella, Veillonella,

Table 1 Study participant characteristics

\begin{tabular}{|c|c|c|c|}
\hline & HIV-infected $N=46$ & HIV-uninfected $N=20$ & $p$ value \\
\hline Age, mean (SD), years & $41(13)$ & $40(10)$ & 0.57 \\
\hline Gender (male), $n$ (\%) & $43(94)$ & $18(90)$ & 0.64 \\
\hline Smoker, n (\%) & $16(35)$ & $9(45)$ & 0.26 \\
\hline \multicolumn{4}{|l|}{ Risk behaviors for HIV infection } \\
\hline MSM, $n(\%)$ & $38(83)$ & NA & ND \\
\hline IDU, $n(\%)$ & $5(11)$ & NA & ND \\
\hline WBC, mean (SD), count/dL & $5424(1623)$ & $5995(1682)$ & 0.20 \\
\hline 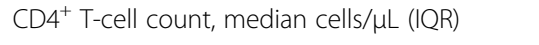 & $438(274-618)$ & ND & ND \\
\hline 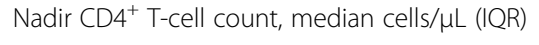 & $164(94-306)$ & ND & ND \\
\hline CD4 $^{+}$T-cell count $\leqq 200$ cells/ $\mu \mathrm{L}, n(\%)$ & $6(13)$ & ND & ND \\
\hline Plasma viral load, median copies/mL (IQR) & $0(0-4505)$ & ND & ND \\
\hline Plasma viral load > 200 copies/mL, $n$ (\%) & $13(28)$ & ND & ND \\
\hline Treatment with ART, $n$ (\%) & $34(74)$ & NA & ND \\
\hline
\end{tabular}

SD standard deviations, MSM men who have sex with men, IDU intravenous drug users, NA not applicable, WBC white blood cell, IQR interquartile range, ND not done, $A R T$ antiretroviral therapy

Age and WBC counts were summarized by means and compared using $t$-tests. Gender and smoking status were compared using Fisher's exact tests 
Neisseria, Fusobacterium, Rothia, Leptotrichia, Actinomyces, Haemophilus, Porphyromonas, Campylobacter, Capnocytophaga, Oribacterium, Granulicatella, Atopobium, Selenomonas) were common to both groups (Fig. 1). Megasphaera and Catonella belonged to the core bacteriome of the HIV-infected group, and Bulleidia and Gemella belonged to that of the HIV-uninfected group. Comparing the relative abundance of the common core bacteriome genera, we found that Neisseria (6\% mean relative abundance for the HIV-infected individuals and $11.2 \%$ for the HIV-uninfected individuals, $p=0.03$ ), Fusobacterium (6.7\% and 9.8\%, respectively, $p=0.02)$ and Haemophilus $(2.9 \%$ and $4.4 \%$, respectively, $p=0.01$ ) were significantly decreased, and Streptococcus $(16.9 \%$ and $13.2 \%$, respectively, $p=0.04$ ) was significantly increased in the HIV-infected group (Fig. 2).

To identify major species-level differences, we searched the species whose core bacteriome colonization rate or relative abundance differed significantly between the groups. Capnocytophaga ochracea, Neisseria cinerea, and Selenomonas noxia colonized the HIV-infected group more frequently, with a higher relative abundance than that in the HIV-uninfected group (Table 2). Veillonella parvula had a similar colonization rate, but its relative abundance was significantly higher in the HIV-uninfected group than that in the HIV-infected group (Table 2).

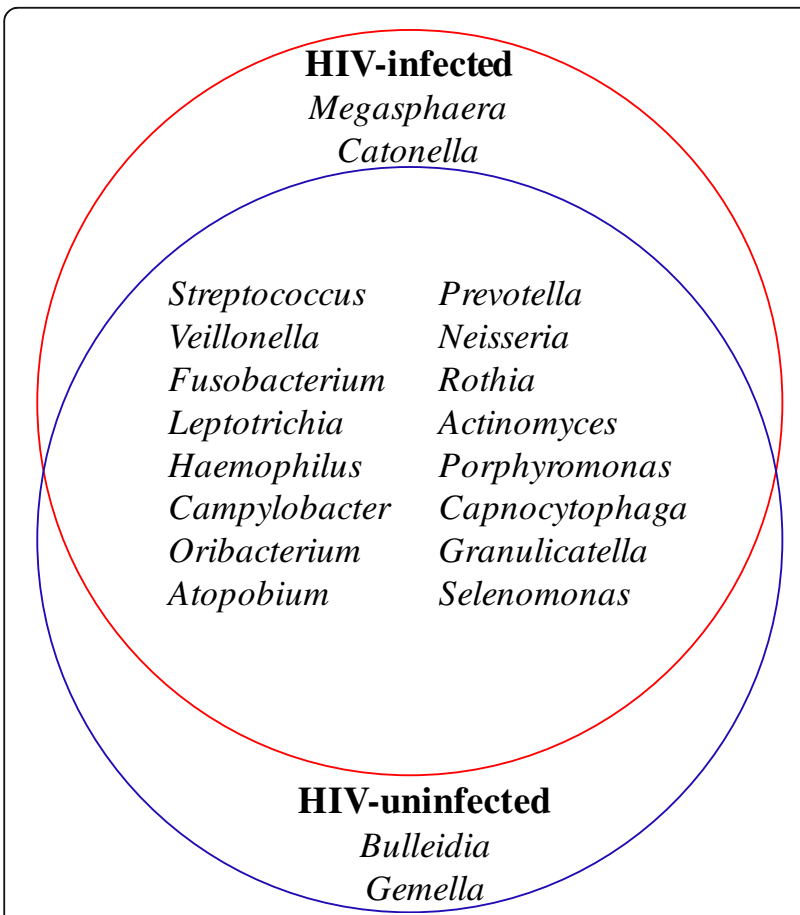

Fig. 1 Palatine tonsil core bacteriome in the HIV-infected and HIV-uninfected individuals
The palatine tonsil mycobiome did not differ between HIV-infected and HIV-uninfected individuals

One HIV-infected sample and three HIV-uninfected samples did not yield sufficient reads for analysis; therefore, $45 \mathrm{HIV}$-infected and $17 \mathrm{HIV}$-uninfected participants were included in the fungal analysis. We first assessed the alpha diversity of the palatine tonsil mycobiome in 52 reads. Unlike the bacteriome, the HIV-infected and HIV-uninfected samples did not significantly differ in terms of alpha diversity (Additional file 1: Table S3). Beta diversity, determined by principal coordinates analysis using weighted UniFrac distance metrics, also did not differ between the two groups $(p=0.47$, PERMANOVA) (Additional file 3: Figure S2).

We compared the relative abundances between the two groups at different taxonomic levels to determine the impact of HIV infection on the palatine tonsil mycobiome.

Phylum-level analysis revealed that two phyla, Ascomycota and Basidiomycota, were abundant, with Ascomycota being the most abundant in both groups (Additional file 1: Table S4). The relative abundances of Ascomycota and Basidiomycota did not significantly differ between the groups. Of the total reads, $1.5 \%$ were classified as unidentified phyla or not classified.

Genus-level analysis identified a total of 43 genera in both groups. The median number of detected genera did not significantly differ between the HIV-infected (4, IQR $4-6)$ and HIV-uninfected individuals (5, IQR 4-6) ( $p=$ 0.6). The six most abundant fungal genera were Candida, Malassezia, Saccharomyces, Debaryomyces, Aspergillus and Penicillium (Fig. 3). Candida and Malassezia colonized the majority of both groups, but no genera colonized $100 \%$ of each group. Only the relative abundance of Penicillium was significantly higher in the HIV-uninfected individuals than that in the HIV-infected individuals among the six most abundant genera.

We used linear discriminant effect size analysis (LEfSe) with default parameters to further identify fungal taxa that were differentially represented between the HIV-infected and HIV-uninfected individuals [26]. LEfSe analysis revealed that the HIV-uninfected group had a significant increase in the order Eurotiales and its family Trichocomaceae, which contains the genera Aspergillus and Penicillium (Additional file 4: Figure S3). No fungal OTUs differed significantly between the groups by LEfSe.

\section{Microbial intercorrelations differed in the HIV-infected individuals}

We determined the correlations between individual genera of the palatine tonsil bacteriome and mycobiome within their respective communities and across the two communities. In the core bacteriome, the correlation pattern differed between the groups. The HIV-infected 


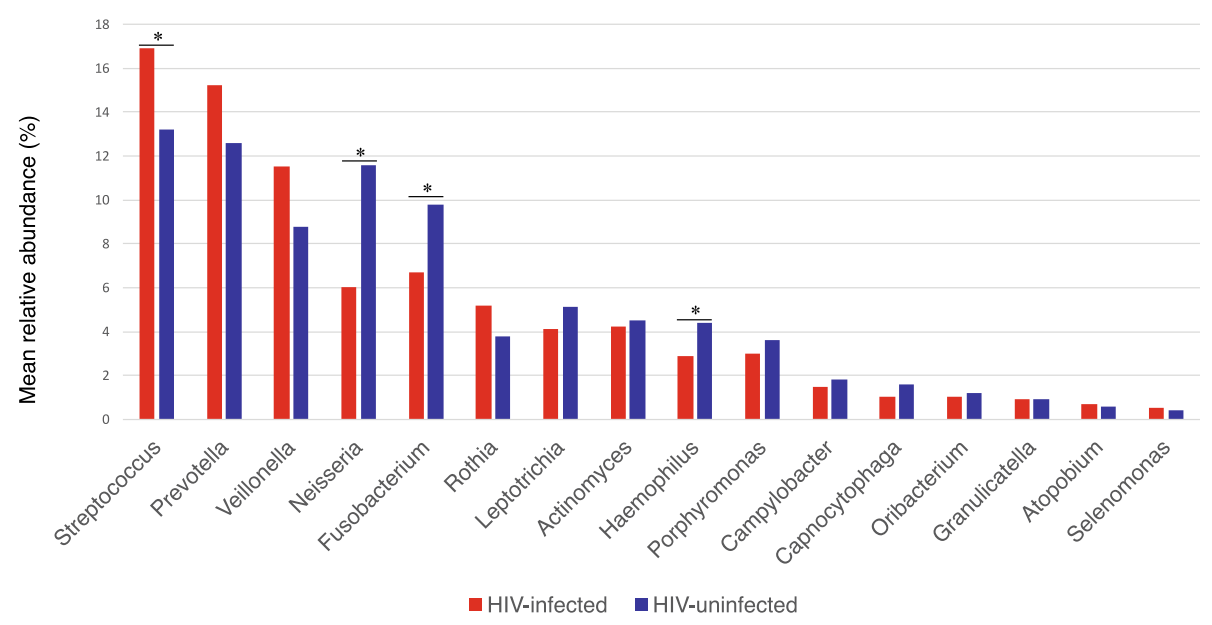

Fig. 2 Relative abundances of the common core bacterial genera in the HIV-infected and HIV-uninfected individuals; ${ }^{*} p<0.05$, Wilcoxon rank-sum test

group had 13 positive bacterial relationships and 9 negative relationships (Additional file 1: Table S5), while the HIV-uninfected group had 7 positive bacterial relationships and 5 negative relationships (Additional file 1: Table S6). In the mycobiome, only HIV-infected group had 1 negative relationship (Candida-Malassezia, rho = $-0.56, p<0.001$ ) among the six most abundant genera. Next, we assessed the correlation between the bacteriome and the mycobiome. The numbers of observed bacterial and fungal OTUs did not correlate with each other in either the HIV-infected (rho $=-0.01, p=0.95$ ) or HIV-uninfected groups (rho $=-0.15, \quad p=0.55$ ) (Additional file 5: Figure S4). The HIV-infected group had no strong correlations ( $r$ ho $>0.5$ or rho $<-0.5$ ), and 3 weak correlations (rho $>0.3$ or rho $<-0.3$ ) between the bacteriome and mycobiome (Additional file 1: Table S7). In comparison, the HIV-uninfected group had 2 strong correlations (Additional file 1: Table S8). Both groups demonstrated negative relationships between Candida and Neisseria and Candida and Capnocytophaga.

\section{HIV immune status did not impact the palatine tonsil microbiome in HIV-infected individuals}

We studied the relationship between $\mathrm{CD} 4^{+} \mathrm{T}$-cell counts and the microbiome in the HIV-infected individuals. $\mathrm{CD} 4^{+} \mathrm{T}$-cell counts were not correlated with the number of observed bacterial (rho $=0.06, p=0.69$ ) or fungal (rho $=0.21, p=0.17$ ) OTUs (Additional file 6: Figure S5). When the HIV-infected individuals were divided into a high CD4 $\left(\mathrm{CD}^{+}{ }^{+}\right.$T-cell count $\geq 350$ cells/ $\left.\mu\right)$ or low CD4 group $\left(\mathrm{CD} 4^{+}\right.$T-cell count $<350$ cells $/ \mu$ ), the two groups did not significantly differ in terms of their alpha diversity of bacteriome (Additional file 1: Table S9) and mycobiome (Additional file 1: Table S10). Beta diversity by principal coordinates analysis using weighted UniFrac distance metrics also did not differ between the two groups (bacteriome, $p=0.76$; mycobiome, $p=0.92$, PERMANOVA). We did not observe any influence of different HIV immune status (ART-treated vs ART-untreated and detectable viremia vs no viremia) on bacteriome and mycobiome in terms of alpha and beta diversity (data not shown). We also compared the microbiome between Men who have sex with men (MSM) and non-MSM and did not observe the difference between them (data not shown).

\section{Discussion}

Our study identified the palatine tonsil core microbiome and showed that the HIV-infected individuals had a different palatine tonsil bacteriome and bacterial intercorrelations than the HIV-uninfected individuals. In contrast, the palatine tonsil mycobiome did not differ significantly between the two groups. The microbial

Table 2 Bacterial species with different presence and relative abundances in the HIV-infected and HIV-uninfected individuals

\begin{tabular}{|c|c|c|c|c|c|c|}
\hline & \multicolumn{3}{|l|}{ Presence (\%) } & \multicolumn{3}{|c|}{ Median relative abundance, \% } \\
\hline & HIV-infected $N=45$ & HIV-uninfected $N=20$ & $p$ value & HIV-infected $N=45$ & HIV-uninfected $N=20$ & $p$ value \\
\hline Capnocytophaga ochracea & $26(58)$ & $1(5)$ & $<0.001$ & 0.01 & 0 & $<0.001$ \\
\hline Neisseria cinerea & $24(53)$ & $4(20)$ & 0.02 & 0.07 & 0 & 0.005 \\
\hline Selenomonas noxia & $28(62)$ & $5(25)$ & 0.007 & 0.01 & 0.0001 & 0.02 \\
\hline Veillonella parvula & $44(98)$ & $19(95)$ & 0.52 & 0.3 & 2.0 & 0.01 \\
\hline
\end{tabular}

Presence was compared using Fisher's exact tests. Relative abundance was summarized by medians and compared using Wilcoxon rank-sum tests 


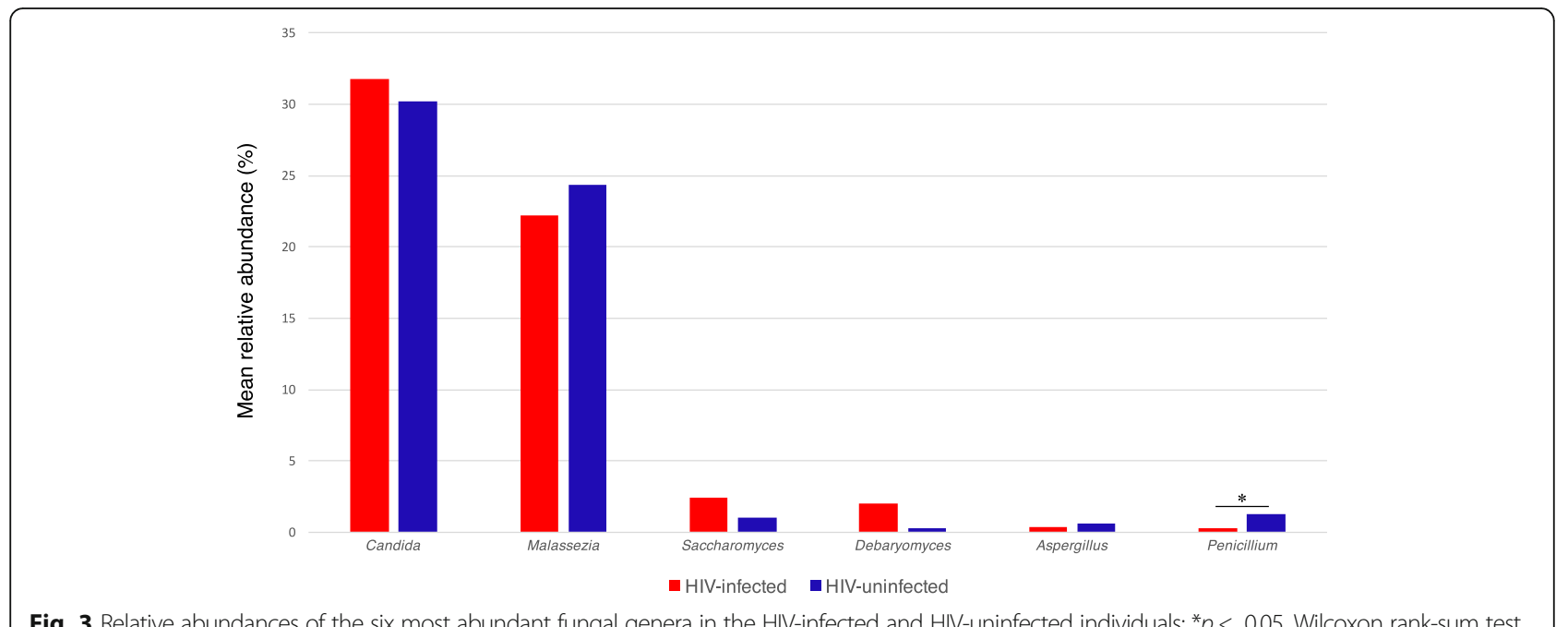

Fig. 3 Relative abundances of the six most abundant fungal genera in the HIV-infected and HIV-uninfected individuals; ${ }^{*} p<0.05$, Wilcoxon rank-sum test

intercorrelations between the bacteriome and mycobiome were relatively similar between the two groups. In the HIV-infected individuals, HIV immune status did not impact the palatine tonsil microbiome, suggesting that HIV infection itself played an important role in palatine tonsil microbial composition.

As with previous studies that analyzed the bacteriome of other specimens from HIV-infected individuals, such as saliva, bronchoalveolar lavage (BAL) and stool $[6,7,13,27]$, the HIV-infected individuals had altered palatine tonsil bacteriome. Changes of alpha diversity in HIV infection are variable, increased in some studies, decreased or not changed in others [20, 27-31]. However, most previous cross-sectional studies detected dysbiotic changes, such as the emergence and increase in pathogenic bacteria and decrease in beneficial bacteria, in HIV-infected individuals [7]. Alterations of bacterial composition in HIV infection differ among organs. We observed increase of Firmicutes and decrease of Proteobacteria in the palatine tonsil. However, previous studies reported inverse results in the gut and the mouth, and others did not note a difference among these phyla in the rectum $[11,15,32]$. This may be because each organ has a specific microbiome and is influenced differently by HIV infection. In the oral microbiome of HIV-infected individuals, the relative abundances of Prevotella, Megasphaera and Campylobacter increased, while that of the normal oral flora Streptococcus decreased [14, 33]. Another study reported that the genus Capnocytophaga is a member of the core oral microbiome in HIV-infected individuals [21] and is associated with AIDS patients with periodontitis [34]. In our study, we observed the genus Capnocytophaga to be the common core microbiome in both the HIV-infected and HIV-uninfected individuals. However, the relative abundance and presence of Capnocytophaga ochracea increased significantly in the palatine tonsil microbiome of the HIV-infected individuals, regardless of immune status. C. ochracea is reported to produce an immunosuppressive factor [35] and to degrade immunoglobulin [36]. These abilities may enhance colonization of this or other opportunistic pathogens and may be involved in the development of opportunistic respiratory infections by modulating the immune system and inducing dysbiosis in HIV-infected individuals. Further research is needed to determine the effect of $C$. ochracea on HIV-infected individuals.

In contrast to the bacteriome, the palatine tonsil mycobiome demonstrated only small compositional differences and did not differ in alpha or beta diversity between the two groups. Few studies have been published on the oral and respiratory mycobiome of HIV-infected individuals [21, 37]. A previous mycobiome research on oral wash samples found that the core mycobiome of HIV-infected individuals was different from that of controls [21]. However, this study compared only some fungal composition and did not compare alpha or beta diversity; therefore, overall mycobiome differences between HIV-infected and HIV-uninfected individuals were unclear. Cryptococcus species and $P$. jirovecii, which are frequent opportunistic pathogens [38-40] and are identified as part of the lung mycobiome in HIV-infected individuals [37], were not detected in the palatine tonsil mycobiome. This result indicated that each organ has a distinct community structure and the palatine tonsil may not be an important organ for opportunistic fungal colonization.

The palatine tonsillar bacterial community in HIV-infected individuals differed in its taxonomic composition as well as its bacterial genera correlation patterns. For example, the negative relationship between the normal commensal flora Neisseria [41] and the pathogenic genus 
Prevotella [42] was lost in HIV-infected individuals; however, Neisseria-Veillonella were negatively correlated and Prevotella-Veillonella were positively correlated in HIV-infected groups. Decreased Neisseria resulted in increased Prevotella and Veillonella in HIV-infected individuals. Increased numbers of Prevotella and Veillonella have been shown to be associated with respiratory inflammation [42]. Another study indicated that increased Prevotella abundance in HIV-infected individuals was related to mucosal and systemic immune activation [32]. Chronic inflammation induced by an altered bacteriome may lead to non-AIDS-defining oral diseases, such as oral squamous cell carcinoma, recurrent aphthous ulcers and necrotizing ulcerative periodontitis, in HIV-infected individuals.

Although there were slight differences, the microbial intercorrelations between the bacteriome and mycobiome were relatively similar between both groups. Both demonstrated negative correlations between Neisseria and Candida and Capnocytophaga and Candida. These results suggest that universal relationships between Candida and symbiotic bacteria may inhibit or support each other within the pharyngeal microbiome. Previous research showed that commensal bacterial species inhibit Candida virulence and growth by preventing biofilm formation and hyphenation [43-46]. In addition, several commensal bacteria have been described to modulate the murine immune system via Th17 cells and Treg cells; these cells are essential for the immune response against Candida [47-49]. In practice, specific medical conditions, such as antibiotic use and intensive care unit stays, may cause human microbiome dysbiosis [50, 51] and increase Candida infections [52]. We speculate that dysbiotic microbial flora and increased candidiasis in HIV-infected individuals may be correlated.

The influence of immune status on the microbiome remains unclear in HIV-infected individuals. A recent study reported that $\mathrm{CD}_{4}{ }^{+} \mathrm{T}$-cell counts do not affect oral or BAL microbiome in HIV-infected individuals [33]. Another study showed that introduction of ART has no influence on the oral microbiome [13]. Despite immune status recovery due to ART, microbiome and metagenomic functions in HIV-infected individuals have been found to differ from those of healthy individuals, suggesting HIV infection itself is important to the microbiome [28, 29, 53]. HIV-infected individuals are more likely to develop cardiovascular disease, lung disease and malignancy, even in the ART era [54-56]. A dysbiotic microbiome is considered to play a prominent role in increased comorbidities in HIV-infected individuals [57].

This study had several limitations. First, it focused on bacterial and fungal communities, but other organisms, such as viruses, archaea and protozoa, may also be factors [58]. Another limitation is that some factors such as diet, risk behaviors and antibiotics use in past months were not controlled in our analysis. Finally, we only included a few HIV-infected individuals with severe immunodeficiency $\left(\mathrm{CD}^{+}{ }^{+} \mathrm{T}\right.$-cell count $<200$ cells $/ \mu \mathrm{L}$ or ART-untreated), due to the exclusion criterion of antibiotic use. Therefore, more HIV-infected individuals with severe immunodeficiency are needed to elucidate the true impact of HIV immune status on the microbiome.

\section{Conclusion}

We conducted a cross-sectional study to describe the palatine tonsil microbiome in HIV-infected individuals and found that the bacteriome and bacterial intercorrelations differed significantly between the HIV-infected and HIV-uninfected individuals. In contrast to the bacteriome, the palatine tonsil mycobiome did not differ significantly between the two groups. The microbial intercorrelations between the bacteriome and mycobiome were relatively similar between both groups. In the HIV-infected individuals, HIV immune status did not impact the palatine tonsil microbiome, suggesting that HIV infection itself played a vital role in the palatine tonsil microbial composition.

\section{Methods}

\section{Participants and sample collection}

During the study period between October 2016 and June 2017, 72 HIV-infected individuals from Toho University Omori Medical Center were initially evaluated. Exclusion criteria were: (a) having acute respiratory symptoms; (b) taking more than five medications besides ART; (c) antibiotics use within the last 4 weeks; (d) history of palatine tonsillectomy; (e) unable to be sampled correctly for anatomical reasons; (f) disagreement to participate. We excluded total $26 \mathrm{HIV}$-infected individuals (14 with [b], 6 with [f], 5 with [c] and 1 with [e]). Forty-six participants were included after meeting the criteria. Additionally, 20 sex- and age-matched healthy HIV-negative individuals were included from the same medical center. We obtained clinical and demographic data by performing standardized subject interviews and medical record reviews. This cross-sectional study protocol was approved by the Institution Ethics Committee of Toho University School of Medicine (Number: A16072_A17082). All participants provided written informed consent in accordance with the Declaration of Helsinki. Palatine tonsil microbiome samples were collected with Catch-All Sample Collection Swabs (Epicentre, Madison, WI, USA) following Human Microbiome Project procedures (http://hmpdacc.org/doc/HMP_MOP_ Version12_0_072910.pdf). Before sampling, mouthwashes 
were not used. Immediately after swabbing, each swab was swirled in $750 \mu \mathrm{L}$ of MoBio buffer in a MoBio tube (MO BIO Laboratories, Inc., Carlsbad, CA, USA) on ice, and the tubes were stored at $-80^{\circ} \mathrm{C}$ until DNA extraction was performed. All specimens were taken by a single doctor to avoid sampling biases.

\section{DNA extraction and sequencing}

All samples, including negative control swab samples, were collected, and genomic DNA (gDNA) was extracted using a PowerSoil DNA Isolation Kit (MO BIO Laboratories, Inc., Carlsbad, CA, USA), with minor modifications [59]. Briefly, samples were heat-lysed at $70{ }^{\circ} \mathrm{C}$ for $10 \mathrm{~min}$ after adding $\mathrm{C} 1$ lysis buffer. The mechanical bead beating step was performed using FastPrep FP120 (Thermo Savant, Carlsbad, CA, USA) for $90 \mathrm{~s}$ at 5.5 speed to enhance chemical, heat, and mechanical lysis. A blank swab was included as a control in the gDNA extraction step to assess possible contamination.

Sequencing libraries were prepared for Illumina MiSeq (Illumina, San Diego, CA, USA). For bacterial analysis, the primer set consisting of 341F (5' -CCTACGGGNGG CWGCAG-3') and 806R (5'-GACTACHVGGGTATCT AATCC-3') was used to target the 16S rRNA V3 and V4 regions [60]. A 16S rRNA library for the Illumina MiSeq platform was prepared per the manufacturer's protocol (http://jp.support.illumina.com/content/dam/illumina-support/documents/documentation/chemistry_documentation/16s/16s-metagenomic-library-prep-guide15044223-b.pdf). In the PCR clean-up step, a Wizard SV Gel and PCR Clean-Up System (Promega, St. Louis, MO, USA) was used to purify PCR amplicons [61]. For fungal analysis, we amplified the internal transcribed spacer (ITS) 1 rDNA region with a primer set consisting of ITS1F (5' -CTTGGTCATTTAGAGGAAGTAA-3') and ITS2 (5' - GCTGCGTTCTTCATCGATGC-3') [21]. DNA was amplified with an initial denaturation step at $95^{\circ} \mathrm{C}$ for $3 \mathrm{~min}$, followed by 40 cycles of denaturation at $95^{\circ} \mathrm{C}$ for $30 \mathrm{~s}$, annealing at $55{ }^{\circ} \mathrm{C}$ for $30 \mathrm{~s}$, and elongation at $72{ }^{\circ} \mathrm{C}$ for $30 \mathrm{~s}$, followed by a final elongation step at $72{ }^{\circ} \mathrm{C}$ for $5 \mathrm{~min}$. Duplicate amplifications were performed for each reaction and mixed before the PCR clean-up step. The subsequent procedure was the same as that used for the bacterial analysis. Each library was sequenced with $2 \times$ 300-bp paired-end reads on a MiSeq system using MiSeq v3 reagent kits (Illumina, San Diego, CA, USA).

\section{Sequence analysis and statistical analysis}

MiSeq sequencing resulted in a total of 4,169,518 reads for the 16S rRNA with a mean of $63,175 \pm 18,146$ sequences per sample and 4,145,030 reads for the ITS1 region with a mean of $62,803 \pm 11,681$ sequences per sample. Sequence data were submitted to DDBJ under the accession number DRA006313.
The sequencing data were processed using CLC Genomic Workbench 10.0.1 and CLC Microbial Genomics Module 2.5 (Qiagen, Redwood City, CA, USA) [62]. The overlapping paired-end reads were merged and trimmed, and chimeric reads were filtered using default parameters. The remaining reads were clustered into OTUs with $97 \%$ identity using the Greengenes database (version 13_5) as the reference for the 16S rRNA data [63] and the UNITE database (version 7.1) as the reference for the ITS data [64]. OTUs with less than 42 reads were removed from bacterial analysis, leaving a total of 511 bacterial OTUs. OTUs annotated to kingdom Plantae and sequences detected in the control were removed from fungal analysis, leaving a total of 238 fungal OTUs.

Alpha diversity was calculated as the number of observed OTUs, Shannon entropy [65] and phylogenetic diversity [66] by CLC Genomic Workbench. Beta diversity was measured as a weighted UniFrac distance based on the OTU table [67] by CLC Genomic Workbench. Relative abundance of the taxa was calculated from an unrarefied OTU table. We used $t$-tests to compare continuous variable means, Fisher's exact tests to compare categorical variable proportions and the Wilcoxon rank-sum test to compare alpha diversity and relative abundance values between the groups using $R$ [68]. PERMANOVA was performed to compare beta diversity using CLC Genomic Workbench. Spearman's correlation tests were computed using $\mathrm{R}$ and illustrated with the qgraph $\mathrm{R}$ package [69]. LEfSe, which is an algorithm for identifying genomic taxa whose relative abundances differ significantly between groups [26], was used in the mycobiome analysis with default parameters. $p$ values < 0.05 were considered to indicate statistical significance. $p$ values were corrected for multiple testing controlling the false discovery rate at analyzing the intercorrelations [70].

\section{Additional files}

Additional file 1: Table S1. Bacterial alpha diversity of the palatine tonsil. Table S2. Bacterial phyla and their differences in relative abundance (mean, \%) between HIV-infected and uninfected individuals in the palatine tonsil. Table S3. Fungal alpha diversity of the palatine tonsil. Table S4. The relative abandance (mean, \%) of fungal phyla in the palatine tonsil. Table S5. Correlations among the common core bacterial genera in HIV-infected individuals. Table S6. Correlations among the common core bacterial genera in HIV-uninfected individuals. Table S7. Correlation between bacteriome and mycobiome in HIV-infected individuals. Table S8. Correlation between bacteriome and mycobiome in HIV-uninfected individuals. Table S9. Bacterial alpha diversity of palatine tonsil in HIV-infected individuals. Table S10. Fungal alpha diversity of palatine tonsil in HIV-infected individuals. (XLSX $21 \mathrm{~kb}$ )

Additional file 2: Figure S1. Principal coordinates analysis plots of bacterial beta diversity using weighted UniFrac distance. The HIV-infected and HIV-uninfected individuals are colored red and blue, respectively. (PDF 50 kb)

Additional file 3: Figure S2. Principal coordinates analysis plots of fungal beta diversity using weighted UniFrac distance. The HIV-infected and HIV-uninfected individuals are colored red and blue, respectively. (PDF $47 \mathrm{~kb}$ ) 
Additional file 4: Figure S3. LEfSe shown as a cladogram (a) and LDA score (b). The HIV-uninfected individuals are displayed in red. (PDF 224 kb)

Additional file 5: Figure S4. Scatter plot showing the relationship between the number of observed bacterial and fungal OTUs in the HIV-infected (a) and HIV-uninfected individuals (b). (PDF 67 kb)

Additional file 6: Figure S5. Scatter plot showing the relationship between CD4+ T-cell counts and the number of observed bacterial (a) and fungal OTUs (b) in the HIV-infected individuals. (PDF 57 kb)

\section{Abbreviations}

AIDS: Acquired immunodeficiency syndrome; ART: Antiretroviral therapy; BAL: bronchoalveolar lavage; gDNA: Genomic DNA; HIV: Human immunodeficiency virus; IQR: Interquartile range; ITS: Internal transcribed spacer; LEfSe: Linear discriminant effect size analysis; MALT: Mucosaassociated lymphoid tissue; MSM: Men who have sex with men; NA: Not applicable; ND: Not done; OTU: Operational taxonomy unit; PERMANOVA: Permutational multivariate analysis of variance; SD: Standard deviations; WBC: White blood cell

\section{Availability of data and materials}

Raw sequences are available in the DDBJ under accession number DRA006313.

\section{Authors' contributions}

YF and KT designed this study. YF performed the sampling. YF, KA and YI performed laboratory analyses. All authors performed data analysis and interpretation of the results. YF wrote this paper. All authors contributed, read and approved the final manuscript.

\section{Ethics approval and consent to participate}

This cross-sectional study protocol was approved by the Institution Ethics Committee of Toho University School of Medicine (Number:

A16072_A17082). All participants provided written informed consent in accordance with the Declaration of Helsinki.

\section{Consent for publication}

Not applicable.

\section{Competing interests}

The authors declare that they have no competing interests.

\section{Publisher's Note}

Springer Nature remains neutral with regard to jurisdictional claims in published maps and institutional affiliations.

\section{Received: 15 March 2018 Accepted: 28 September 2018} Published online: 05 October 2018

\section{References}

1. Pantaleo G, Graziosi C, Fauci AS. The immunopathogenesis of human immunodeficiency virus infection. N Engl J Med. 1993;328:327-35.

2. Huang L, Quartin A, Jones D, Havlir DV. Intensive care of patients with HIV infection. N Engl J Med. 2006;355:173-81.

3. Lundgren JD, Babiker AG, Gordin F, Emery S, Grund B, Sharma S, et al. Initiation of antiretroviral therapy in early asymptomatic HIV infection. N Engl J Med. 2015:373:795-807.

4. Yin $Z$, Rice $B D$, Waight $P$, Miller $E$, George $R$, Brown AE, et al. Invasive pneumococcal disease among HIV-positive individuals, 2000-2009. AIDS. 2012;26:87-94.

5. Guaraldi G, Orlando G, Zona S, Menozzi M, Carli F, Garlassi E, et al. Premature age-related comorbidities among HIV-infected persons compared with the general population. Clin Infect Dis. 2011;53:1120-6.

6. Moyes DL, Saxena D, John MD, Malamud D. The gut and oral microbiome in HIV disease: a workshop report. Oral Dis. 2016;22(Suppl 1):166-70.

7. Williams B, Landay A, Presti RM. Microbiome alterations in HIV infection a review. Cell Microbiol. 2016;18:645-51.

8. Peterson J, Garges S, Giovanni M, Mclnnes P, Wang L, Schloss JA, et al. The $\mathrm{NIH}$ human microbiome project. Genome Res. 2009;19:2317-23.
9. Twigg HL 3rd, Weinstock GM, Knox KS. Lung microbiome in human immunodeficiency virus infection. Transl Res. 2017;179:97-107.

10. Liu J, Williams B, Frank D, Dillon SM, Wilson CC, Landay AL. Inside out: HIV the gut microbiome, and the mucosal immune system. J Immunol. 2017; 198:605-14.

11. Nowak RG, Bentzen SM, Ravel J, Crowell TA, Dauda W, Ma B, et al. Rectal microbiota among HIV-uninfected, untreated HIV, and treated HIV-infected in Nigeria. AIDS. 2017;31:857-62.

12. Routy JP, Mehraj V. Potential contribution of gut microbiota and systemic inflammation on HIV vaccine effectiveness and vaccine design. AIDS Res Ther. 2017;14:48

13. Presti RM, Handley SA, Droit L, Ghannoum M, Jacobson M, Shiboski CH, et al. Alterations in the oral microbiome in HIV-infected participants after antiretroviral therapy administration are influenced by immune status. AIDS. 2018:32:1279-87.

14. Dang AT, Cotton S, Sankaran-Walters S, Li CS, Lee CY, Dandekar S, et al. Evidence of an increased pathogenic footprint in the lingual microbiome of untreated HIV infected patients. BMC Microbiol. 2012;12:153.

15. Yang L, Poles MA, Fisch GS, Ma Y, Nossa C, Phelan JA, et al. HIV-induced immunosuppression is associated with colonization of the proximal gut by environmental bacteria. AIDS. 2016:30:19-29.

16. Buve A, Jespers V, Crucitti T, Fichorova RN. The vaginal microbiota and susceptibility to HIV. AIDS. 2014;28:2333-44.

17. Liu CM, Prodger JL, Tobian AAR, Abraham AG, Kigozi G, Hungate BA, et al. Penile anaerobic Dysbiosis as a risk factor for HIV infection. MBio. 2017;8(4)

18. Klatt NR, Cheu R, Birse K, Zevin AS, Perner M, Noel-Romas L, et al. Vaginal bacteria modify HIV tenofovir microbicide efficacy in African women. Science. 2017:356:938-45.

19. Lozupone CA, Rhodes ME, Neff CP, Fontenot AP, Campbell TB, Palmer BE. HIV-induced alteration in gut microbiota: driving factors, consequences, and effects of antiretroviral therapy. Gut Microbes. 2014;5:562-70.

20. Nowak P, Troseid M, Avershina E, Barqasho B, Neogi U, Holm K, et al. Gut microbiota diversity predicts immune status in HIV-1 infection. AIDS. 2015; 29:2409-18.

21. Mukherjee PK, Chandra J, Retuerto M, Sikaroodi M, Brown RE, Jurevic R, et al Oral mycobiome analysis of HIV-infected patients: identification of Pichia as an antagonist of opportunistic fungi. PLoS Pathog. 2014;10:e1003996.

22. Cui L, Morris A, Ghedin E. The human mycobiome in health and disease. Genome Med. 2013;5:63

23. Perry M, Whyte A. Immunology of the tonsils. Immunol Today. 1998;19:414-21.

24. Jensen A, Fago-Olsen $\mathrm{H}$, Sorensen $\mathrm{CH}$, Kilian M. Molecular mapping to species level of the tonsillar crypt microbiota associated with health and recurrent tonsillitis. PLoS One. 2013;8:e56418.

25. Turnbaugh PJ, Ley RE, Hamady M, Fraser-Liggett CM, Knight R, Gordon II. The human microbiome project. Nature. 2007:449:804-10.

26. Segata N, Izard J, Waldron L, Gevers D, Miropolsky L, Garrett WS, et al. Metagenomic biomarker discovery and explanation. Genome Biol. 2011;12:R60.

27. Noguera-Julian M, Guillen Y, Peterson J, Reznik D, Harris EV, Joseph SJ, et al. Oral microbiome in HIV-associated periodontitis. Medicine (Baltimore). 2017; 96:e5821.

28. Lozupone CA, Li M, Campbell TB, Flores SC, Linderman D, Gebert MJ, et al. Alterations in the gut microbiota associated with HIV-1 infection. Cell Host Microbe. 2013;14:329-39.

29. Twigg HL 3rd, Knox KS, Zhou J, Crothers KA, Nelson DE, Toh E, et al. Effect of advanced HIV infection on the respiratory microbiome. Am J Respir Crit Care Med. 2016;194:226-35.

30. Vujkovic-Cvijin I, Dunham RM, Iwai S, Maher MC, Albright RG, Broadhurst MJ, et al. Dysbiosis of the gut microbiota is associated with HIV disease progression and tryptophan catabolism. Sci Transl Med. 2013;5:193ra91.

31. Dinh DM, Volpe GE, Duffalo C, Bhalchandra S, Tai AK, Kane AV, et al. Intestinal microbiota, microbial translocation, and systemic inflammation in chronic HIV infection. J Infect Dis. 2015;211:19-27.

32. Dillon SM, Lee EJ, Kotter CV, Austin GL, Dong Z, Hecht DK, et al. An altered intestinal mucosal microbiome in HIV-1 infection is associated with mucosal and systemic immune activation and endotoxemia. Mucosal Immunol. 2014;7:983-94.

33. Beck JM, Schloss PD, Venkataraman A, Twigg H 3rd, Jablonski KA, Bushman FD, et al. Multicenter comparison of lung and Oral microbiomes of HIVinfected and HIV-uninfected individuals. Am J Respir Crit Care Med. 2015. 192:1335-44. 
34. Zhang F, He S, Jin J, Dong G, Wu H. Exploring salivary microbiota in AIDS patients with different periodontal statuses using 454 GS-FLX titanium pyrosequencing. Front Cell Infect Microbiol. 2015;5:55.

35. Ochiai K, Senpuku H, Kurita-Ochiai T. Purification of immunosuppressive factor from Capnocytophaga Ochracea. J Med Microbiol. 1998;47:1087-95.

36. Jansen HJ, van der Hoeven JS, van den Kieboom CW, Goertz JH, Camp PJ, Bakkeren JA. Degradation of immunoglobulin $\mathrm{G}$ by periodontal bacteria. Oral Microbiol Immunol. 1994;9:345-51.

37. Cui L, Lucht L, Tipton L, Rogers MB, Fitch A, Kessinger C, et al. Topographic diversity of the respiratory tract mycobiome and alteration in HIV and lung disease. Am J Respir Crit Care Med. 2015;191:932-42.

38. Jarvis JN, Harrison TS. HIV-associated cryptococcal meningitis. AIDS. 2007;21: 2119-29.

39. Buchacz K, Baker RK, Palella FJ Jr, Chmiel JS, Lichtenstein KA, Novak RM, et al. AIDS-defining opportunistic illnesses in US patients, 1994-2007: a cohort study. AIDS. 2010;24:1549-59.

40. Farhour Z, Mehraj V, Chen J, Ramendra R, Lu H, Routy JP. Use of (1-->3)beta-d-glucan for diagnosis and management of invasive mycoses in HIVinfected patients. Mycoses. 2018;61(10):718-22.

41. Liu G, Tang CM, Exley RM. Non-pathogenic Neisseria: members of an abundant, multi-habitat, diverse genus. Microbiology. 2015;161:1297-312.

42. Segal LN, Alekseyenko AV, Clemente JC, Kulkarni R, Wu B, Gao Z, et al. Enrichment of lung microbiome with supraglottic taxa is associated with increased pulmonary inflammation. Microbiome. 2013;1:19.

43. Bachtiar EW, Bachtiar BM, Jarosz LM, Amir LR, Sunarto $H$, Ganin $\mathrm{H}$, et al. Al-2 of Aggregatibacter actinomycetemcomitans inhibits Candida albicans biofilm formation. Front Cell Infect Microbiol. 2014:4:94

44. Barbosa JO, Rossoni RD, Vilela SF, de Alvarenga JA, Velloso Mdos S, Prata MC, et al. Streptococcus mutans can modulate biofilm formation and attenuate the virulence of Candida albicans. PLoS One. 2016;11:e0150457.

45. Ishijima SA, Hayama K, Burton JP, Reid G, Okada M, Matsushita Y, et al. Effect of Streptococcus salivarius K12 on the in vitro growth of Candida albicans and its protective effect in an oral candidiasis model. Appl Environ Microbiol. 2012;78:2190-9.

46. Matsubara VH, Wang Y, Bandara HM, Mayer MP, Samaranayake LP. Probiotic lactobacilli inhibit early stages of Candida albicans biofilm development by reducing their growth, cell adhesion, and filamentation. Appl Microbiol Biotechnol. 2016:100:6415-26.

47. Atarashi K, Tanoue T, Oshima K, Suda W, Nagano Y, Nishikawa H, et al. Treg induction by a rationally selected mixture of clostridia strains from the human microbiota. Nature. 2013;500:232-6.

48. Farkas AM, Panea C, Goto Y, Nakato G, Galan-Diez M, Narushima S, et al. Induction of Th17 cells by segmented filamentous bacteria in the murine intestine. J Immunol Methods. 2015:421:104-11.

49. Romani L. Immunity to fungal infections. Nat Rev Immunol. 2011;11:275-88.

50. Abeles SR, Jones MB, Santiago-Rodriguez TM, Ly M, Klitgord N, Yooseph S, et al. Microbial diversity in individuals and their household contacts following typical antibiotic courses. Microbiome. 2016;4:1-12.

51. Rogers MB, Firek B, Shi M, Yeh A, Brower-Sinning R, Aveson V, et al. Disruption of the microbiota across multiple body sites in critically ill children. Microbiome. 2016:4:66.

52. Pfaller MA, Diekema DJ. Epidemiology of invasive candidiasis: a persistent public health problem. Clin Microbiol Rev. 2007;20:133-63.

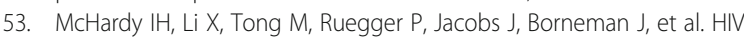
infection is associated with compositional and functional shifts in the rectal mucosal microbiota. Microbiome. 2013;1:26.

54. Sudano I, Spieker LE, Noll G, Corti R, Weber R, Luscher TF. Cardiovascular disease in HIV infection. Am Heart J. 2006;151:1147-55.

55. Fitzpatrick M, Brooks JT, Kaplan JE. Epidemiology of HIV-associated lung disease in the United States. Semin Respir Crit Care Med. 2016;37:181-98.

56. Rubinstein PG, Aboulafia DM, Zloza A. Malignancies in HIV/AIDS: from epidemiology to therapeutic challenges. AIDS. 2014;28:453-65.

57. Vazquez-Castellanos JF, Serrano-Villar S, Latorre A, Artacho A, Ferrus ML, Madrid N, et al. Altered metabolism of gut microbiota contributes to chronic immune activation in HIV-infected individuals. Mucosal Immunol. 2015;8:760-72.

58. Wade WG. The oral microbiome in health and disease. Pharmacol Res. 2013; 69:137-43.

59. Leung MHY, Chan KCK, Lee PKH. Skin fungal community and its correlation with bacterial community of urban Chinese individuals. Microbiome. 2016:4:1-15.
60. Klindworth A, Pruesse E, Schweer T, Peplies J, Quast C, Horn M, et al. Evaluation of general 165 ribosomal RNA gene PCR primers for classical and next-generation sequencing-based diversity studies. Nucleic Acids Res. 2013:41:e1.

61. Jiang H, Barker SC, Shao R. Substantial variation in the extent of mitochondrial genome fragmentation among blood-sucking lice of mammals. Genome Biol Evol. 2013;5:1298-308.

62. Szczepaniak Z, Czarny J, Staninska-Pieta J, Lisiecki P, Zgola-Grzeskowiak A, Cyplik P, et al. Influence of soil contamination with PAH on microbial community dynamics and expression level of genes responsible for biodegradation of PAH and production of rhamnolipids. Environ Sci Pollut Res Int. 2016;23:23043-56.

63. DeSantis TZ, Hugenholtz P, Larsen N, Rojas M, Brodie EL, Keller K, et al. Greengenes, a chimera-checked 16S rRNA gene database and workbench compatible with ARB. Appl Environ Microbiol. 2006;72:5069-72.

64. Kõljalg U, Nilsson RH, Abarenkov K, Tedersoo L, Taylor AFS, Bahram M, et al. Towards a unified paradigm for sequence-based identification of fungi. Mol Ecol. 2013;22:5271-7.

65. Shannon CE. A mathematical theory of communication. Bell Syst Tech J. 1948;27:379-423.

66. Faith DP, Baker AM. Phylogenetic diversity (PD) and biodiversity conservation: some bioinformatics challenges. Evol Bioinformatics Online. 2006;2:121-8

67. Lozupone C, Lladser ME, Knights D, Stombaugh J, Knight R. UniFrac: an effective distance metric for microbial community comparison. ISME J. 2011;5:169-72.

68. Team RC. A language and environment for statistical computing. Vienna: $R$ Foundation for Statistical Computing; 2017.

69. Epskamp S, Cramer AO, Waldorp L, Schmittmann VD, Borsboom D. qgraph: network visualizations of relationships in psychometric data. J Stat Softw. 2012:48:1-18

70. Benjamini Y, Hochberg Y. Controlling the false discovery rate: a practical and powerful approach to multiple testing. J R Stat Soc Ser B Methodol. 1995;57:289-300.

\section{Ready to submit your research? Choose BMC and benefit from:}

- fast, convenient online submission

- thorough peer review by experienced researchers in your field

- rapid publication on acceptance

- support for research data, including large and complex data types

- gold Open Access which fosters wider collaboration and increased citations

- maximum visibility for your research: over $100 \mathrm{M}$ website views per year

At $\mathrm{BMC}$, research is always in progress.

Learn more biomedcentral.com/submission 